\title{
Topology optimization considering body forces
}

\author{
Bin Zheng ${ }^{1}$, Ching-jui Chang ${ }^{2}$, Hae Chang Gea ${ }^{2, a}$ \\ ${ }^{1}$ School of Mechatronics Engineering, University of Electronic Science and Technology of China, Chengdu, 610054, China \\ ${ }^{2}$ Department of Mechanical and Aerospace Engineering, Rutgers University, Piscataway, New Jersey 08854, USA
}

Received 05 September 2008, Accepted 07 January 2009

\begin{abstract}
In this paper, topology optimization problems with three types of body forces are considered: gravitational force, centrifugal force and inertia force. All these body forces are design dependent as they vary with the redistribution of materials during topology optimization process. In this study, a total mean compliance formulation is used to produce the stiffest structure under both external and body forces. Sensitivity analysis is carried out by the adjoint method. Finally, design examples are presented and compared to show the effects of body forces on the optimized results.
\end{abstract}

Key words: Topology optimization, Body forces, Sensitivity analysis

\section{Introduction}

Since Bendsøe and Kikuchi [1] studied topology optimization problem using the homogenization method in 1988, topology optimization has been applied to a variety of structural design problems. Following the traditional topology optimization formulation, boundary conditions of structure design problems including supports and applied loading must be given a priori, and they are kept invariant during the topology optimization procedure. However, for many practical engineering problems, such as pressure loadings and body forces, the boundary conditions is design dependent, that cannot be fully determined until the optimal solution is reached. The special characteristics of design dependence make the application of traditional topology optimization approaches very difficult. Some attempts to solve design dependent topology optimization has been reported. Topology optimization problems under incomplete supporting conditions were studied by Chickermane et al. [2] and Bhul [3]. Topology optimization with pressure loadings has been investigated by Hammer and Olhoff [4], Chen and Kikuchi [5], Fuchs and Shemesh [6], Zheng and Gea [7], and Sigmud and Clausen [8].

Another important class of design dependent loading applications is on structures with body forces, such as gravitational force, centrifugal force and inertia force. They are very important to many mechanical or civil engineering applications. However, researches on the optimal structures with body forces are mostly on the structural profiles and publications of topology optimization with body forces are very limited. Optimal design under self-weight was first studied by Rozvany [9] for plastic design. Karihaloo and Hemp [10] presented the maximum strength/stiffness design of structure members in presence of self-weight by analytical method. Chern and Prager [11] reported the optimal rotating disk in terms of uniform strength for given radial displacement of edge. Huang and Fadel [12] applied shape optimization techniques to the heterogeneous flywheel design. Turteltaub and Washabaugh [13] took into account body force when they studied optimal distribution of material properties for an elastic continuum. Park et al. [14] studied the topology optimization of primary mirror of camera under self-weight. Bruyneel and Duysinx [15] compared the efficiency of different methods used to solve the topology optimization problems under self-weight.

In this paper, topology optimization problems with three types of body forces are considered: gravitational force, centrifugal force and inertia force. For structural design under both external and body forces, a total mean compliance formulation is used to produce the stiffest structure. This paper is arranged as follows: topology problem formulation with body force is described in the next section. Then, sensitivity analysis of mean compliance is discussed. Finally, four design examples are presented and compared.

\section{Problem formulation}

In a traditional topology optimization formulation, applied loads remain invariant even when structural topology changes. However, when body forces are included in the topology optimization formulation, the force term must be changing along with the changes of structural topology. As two schematic centrifugal force examples shown in Fig.1, body forces $\boldsymbol{F}_{b}$ are totally different between these two designs because of the mass distribution difference, even they are rotating around axis $z$ at the same speed $\omega$. Therefore, in 2 order to accurately formulate topology optimization problems with body forces, the applied forces cannot be treated as constant.

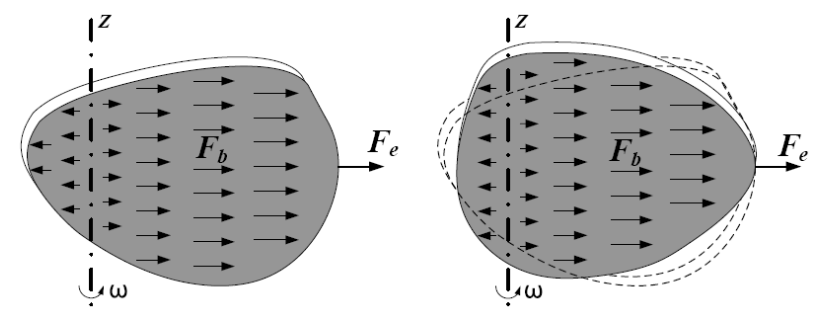

Fig.1. Schematic examples of centrifugal forces

${ }^{\text {a }}$ Corresponding author: gea@ rutgers.edu 
Body force is an external force acting throughout the mass of a structure. Three types of body forces are considered in this paper: gravitational force $\boldsymbol{F}^{G}$, centrifugal force $\boldsymbol{F}^{C}$, and inertial force $\boldsymbol{F}^{I}$. Considering the effective $\rho_{p}$ of the porous material is linearly proportional to its volume density $x_{p}$ as:

$$
\rho_{p}=x_{p} \rho_{0}
$$

where $\rho_{0}$ is the density of the base material.

Then the gravitational force at a point $p$ can be represented as:

$$
\boldsymbol{F}_{p}^{G}=m \boldsymbol{g}=\left(\rho_{p} v_{p}\right) \boldsymbol{g}=x_{p} \rho_{0} v_{p} \boldsymbol{g}
$$

where $\boldsymbol{g}$ is gravitational constant and $v_{p}$ is the volume of the element. Similarly, the centrifugal force and inertial force are defined as:

$$
\boldsymbol{F}_{p}^{C}=x_{p} \rho_{0} v_{p} \omega^{2} r_{p}
$$

and

$$
\boldsymbol{F}_{p}^{I}=-x_{p} \rho_{0} v_{p} \boldsymbol{a}
$$

where $\omega$ is the angular velocity and rp denotes the rotational radius, $\boldsymbol{a}$ is the acceleration of this point. From above three equations, we found all of these body forces are the functions of design variable $x_{p}$. This implies that body forces are design dependent loads.

Topology optimization problem for the stiffest structure can be formulated as to minimize the total mean compliance with a material volume constraint as follows:

$$
\begin{array}{ll}
\min _{x} & \Pi \\
\text { s.t. } & V(x)=\int_{\Omega} x d v \leq \bar{V} \\
& 0<x<1,
\end{array}
$$

where $x$ represents the distribution of volume density in whole design domain; $\bar{V}$ is a predetermined upper bound of volume constraint. Finite Element Method is usually used to determine the relation between the forces and displacement during topology optimization process. Hence the stiffest structure problem formulated by Eq.(5) is transformed to the following numerical expression

$$
\begin{array}{ll}
\min _{x} & \Pi=\boldsymbol{U}^{\boldsymbol{T}} \boldsymbol{K} \boldsymbol{U} \\
\text { s.t. } & V(x)=\sum_{i=1}^{N} x_{i} v_{i} \leq \bar{V} \\
& 0<x_{i} \leq 1, i \in[1, N],
\end{array}
$$

where $x_{i}$ is the design variable of $i$ th finite element cell; $\boldsymbol{K}$ is the global stiffness matrix; $\boldsymbol{U}$ is the displacement vector, $N$ is the total number of the finite element cells, superscript $T$ denotes the transpose operation. To solve the problem using a gradient based method, sensitivity analysis is needed and will be discussed in the next section.

\section{Sensitivity analysis}

Sensitivity analysis provides the search directions during optimization process. In this paper, the adjoint method is utilized to derive the sensitivity of the objective function $\Pi$. First, a new function $\Pi^{*}$ is constructed as

$$
\Pi^{*}=\boldsymbol{U}^{T} \boldsymbol{K} \boldsymbol{U}+\lambda^{T}(\boldsymbol{K} \boldsymbol{U}-\boldsymbol{F}) .
$$

For a discrete model in finite element method, the relation of $\mathbf{K U}=\mathbf{F}$ is always satisfied at static equilibrium condition. Hence, the value of $\Pi^{*}$ is the same as original function $\Pi$ regardless the choice of $\lambda$. In here, $\lambda$ is referred as the adjoint displacement field.

By taking a first-order derivative on both sides of Eq.(7), the sensitivity of $\Pi^{*}$ becomes:

$$
\begin{aligned}
\frac{\partial \Pi^{*}}{\partial x_{i}}= & (\boldsymbol{U}+\lambda)^{T} \frac{\partial \boldsymbol{K}}{\partial x_{i}} \boldsymbol{U} \\
& +(2 \boldsymbol{U}+\lambda)^{T} \frac{\partial \boldsymbol{K}}{\partial x_{i}} \boldsymbol{U}-\lambda^{T} \frac{\partial \boldsymbol{F}}{\partial x_{i}} .
\end{aligned}
$$

In order to avoid the high computational cost of $\partial \boldsymbol{U} / \partial x_{i}$, the arbitrary adjoint displacement vector is set as $\lambda=-2 \boldsymbol{U}$. In this way, all terms which include $\partial \boldsymbol{U} / \partial x_{i}$ in Eq.(8) are cancelled and the sensitivity of $\Pi^{*}$ is reduced to the following form:

$$
\frac{\partial \Pi^{*}}{\partial x_{i}}=-\boldsymbol{U}^{T} \frac{\partial \boldsymbol{K}}{\partial x_{i}} \boldsymbol{U}+2 \boldsymbol{U}^{T} \frac{\partial \boldsymbol{F}}{\partial x_{i}} .
$$

Using a spherical micro-inclusion model in topology optimization (Gea [16]), the effective Young's modulus, $E_{p}$, can be written as

$$
E_{p}=f\left(x_{p}\right) E_{0}, \quad 0 \leq x_{p} \leq 1
$$

where $E_{0}$ is the Young's modulus of base material. Since the first term at the RHS of Eq.(9) can be obtained easily, our attention is focused on the second term.

The applied forces $\boldsymbol{F}$ should include the external force $\boldsymbol{F}_{\mathrm{e}}$ as well as the body forces, $\boldsymbol{F}^{G}, \boldsymbol{F}^{C}$ and $\boldsymbol{F}^{I}$. The external force is normally considered as constant and its derivative with respect to design variables is zero. For the derivatives of gravitational force and centrifugal force, we can take derivatives on both sides of Eq.(2) and (3) as:

$$
\frac{\partial \boldsymbol{F}^{G}}{\partial x_{i}}=\frac{\partial \rho_{i} v_{i} \boldsymbol{g}}{\partial x_{i}}=\rho_{0} v_{i} \boldsymbol{g},
$$




$$
\frac{\partial \boldsymbol{F}^{C}}{\partial x_{i}}=\frac{\partial \rho_{i} v_{i} \omega^{2} r_{i}}{\partial x_{i}}=\rho_{0} v_{i} \omega^{2} r_{i} \text {. }
$$

Since the inertia force may come from linear acceleration or angular acceleration, we need to discuss them separately. If the inertia force is caused by linear acceleration a, then its sensitivity is similar to that of gravitational and centrifugal body forces as:

$$
\frac{\partial \boldsymbol{F}^{I}}{\partial x_{i}}=-\frac{\partial \rho_{i} v_{i} \boldsymbol{a}}{\partial x_{i}}=-\rho_{0} v_{i} \boldsymbol{a}
$$

On the other hand, if the inertia force is caused by a constant angular acceleration $\alpha$, the driving torque $\boldsymbol{T}$ becomes a design dependent variable due to its relation with the moment of inertia $I$ as:

$$
\boldsymbol{T}=I \alpha,
$$

where $\mathrm{T}$ is external torque and $\mathrm{I}$ is the moment of inertia as:

$$
I=\sum_{i=1}^{N} x_{i} \rho_{0} v_{i} r_{i}^{2}
$$

where $r_{i}$ represents the rotational radius of the ith cell. We will use one example to illustrate this case in the next section. Once the sensitivity analyses of body forces are obtained, we can apply the Generalized Convex Approximation method [17] to solve topology optimization problem considering body forces.

\section{Numerical examples}

\subsection{Example 1: Beam under self weight}

The first example is to find the stiffest design of a beam clamped at one end and under a given external traction force, as shown in Fig.2(a). A 30 by 60 finite element model is built, the upper bound of volume constraint is set to be $40 \%$ of total volume. If the self-weight of the structure itself is not considered, the topology optimization result is shown as Fig.2(b). The width of this beam keeps the same except the part near the traction force. When the self-weight is considered, the final optimal result is shown in Fig.2(d). The width of the profile of the structure gradually decreases from the top to the bottom of the design domain as well as the analytical solution shown in Fig.2(c) which is given by Karihaloo and Hemp[10]. The half width of the shape $a(x)$ at the position $\mathrm{x}$ describes the shape of the analytic solution, and it is expressed as

$$
\begin{aligned}
& a(x)= \\
& (T / \gamma) \ln (1+\bar{V}) \gamma / T) \exp [\ln (1+\bar{V}) \gamma / T) x]
\end{aligned}
$$

where

$$
T=F_{t} / E_{0}, \gamma=\rho_{0} g / E, \int_{0}^{1} a(\eta) d \eta=\bar{V} .
$$

Although analytical solution is provided for this optimization problem, most engineering problems considering body forces are not as simple as this one, their analytical solutions always cannot be found. Hence topology optimization method becomes very valuable to solve this type of problem.

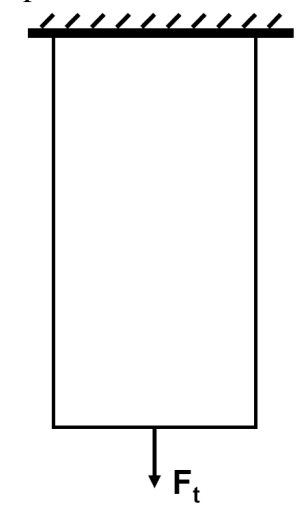

(a) Design domain

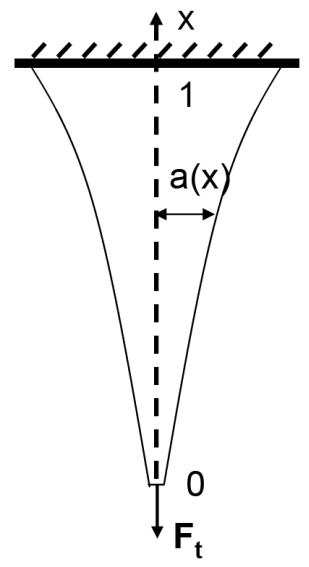

(c) Analytical solution

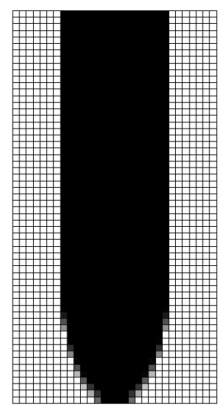

(b) Not considering gravitational force

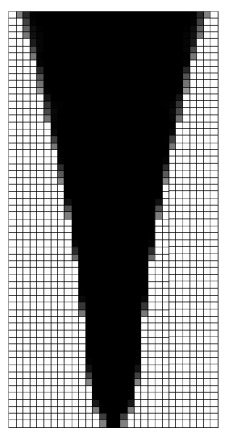

(d) Considering gravitational force
Fig.2. Optimization design for hanging structure considering gravitational force

\subsection{Example 2: Supporting Bracket with Linear Inertia Force}

The second example is to show the effects from linear inertia force using a supporting bracket with linear inertia force. Two forces with the equal magnitude are applied at two top corners of the bracket and two bottom corners have fixed supports as shown in Fig.3. To demonstrate the effect from the inertia forces, the magnitude of external forces are comparable to that of the total inertia forces. Material volume used to design is set to be less than $50 \%$ of total volume. The design domain is discretized by 30 by 60 finite elements.

When the structure does not move, the optimal result is shown in Fig.4(a), and all permitted volume of material is used to generate the support structure. However, when the structure moves with an upward acceleration a, downward inertia forces are applying on all mass points in the design domain. The optimal result is shown in Fig.4(b). More 
material are distributed to the areas near the two fixed supports as because of the additional inertial force.

The most interesting thing is the mass volume used to design the structure in Fig.4(b) is only $46 \%$ of total volume, less than the upper bound of volume constraint. It means the volume constraint is not active for this case. The reason is there exists a compromise between two conflicting trends: 1) By adding as more material as possible to enhance the support structure, the work done by the external force will decreases; 2) The additional material will bring more inertia forces, then the total work done by the inertia force will increase. It is different from the traditional case in which more material means stronger optimal structure.

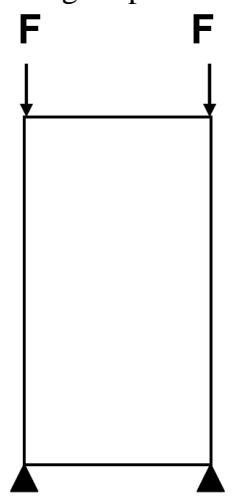

Fig.3. Design domain

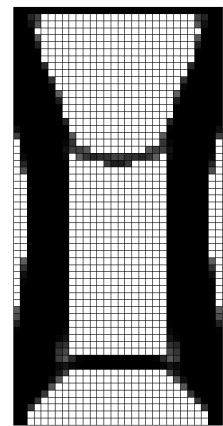

(a)

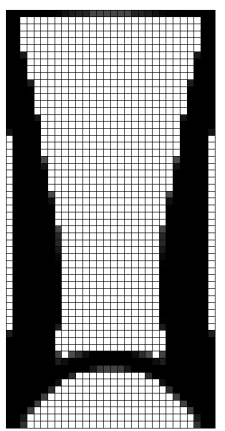

(b)
Fig.4. Optimized structures with and without considering body forces

\subsection{Example 3: Four-leaf Fan}

In this example, a cyclic-symmetric four-leaf rotating fan with the rotating axis perpendicular to the plane of design domain is studied. As we discussed in the section of sensitivity analysis, if the moments of external forces are balanced, the whole structure will be in equilibrium state and have a constant rotation speed. On the other hand, if the moments are not balanced, the whole structure will be moving with an angular acceleration. We will study both cases in this example.

A schematic of design domain is shown in Fig.5(a). A support is placed at the center of the design domain, and this support limits all degree of freedom except rotation one in the design domain plane. The rotation direction of the fan is set as counterclockwise. The driving forces $\boldsymbol{F}_{d}$ are applied at the eight black points around the center, as shown in Fig.5(b).
The resistances on the leaves are simplified as four point loads $\boldsymbol{F}_{t}$ applied at the corners. The entire design domain is separated into A,B,C,D four parts. Since a cyclic-symmetric structure is designed, the four areas have the same support and loading boundary conditions. We only need to design the structure in area A, whose details are shown in Fig.5(b), then the whole structure can be assembled. A 50 by 50 finite elements model is constructed in area A. The material is limited to $30 \%$ of the total volume.

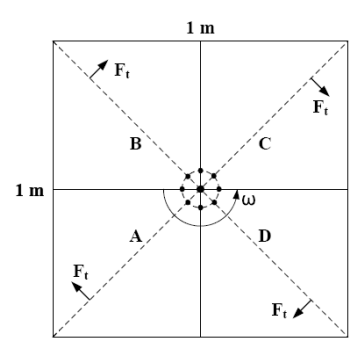

(a) Design domain for a four-leaf revolving fan

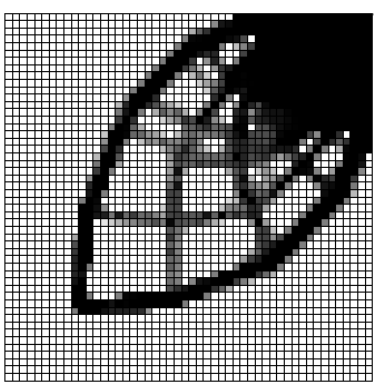

(a) Optimized single leaf without rotation

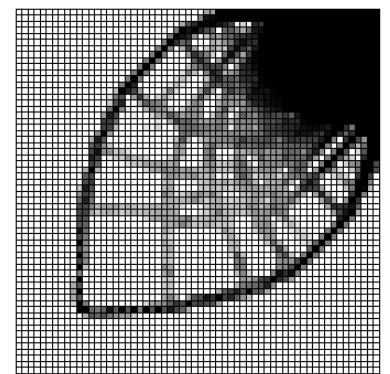

(c) Optimized single leaf with $\alpha=1 \mathrm{rad} / \mathrm{s}^{2}, \omega=0 \mathrm{rad} / \mathrm{s}$

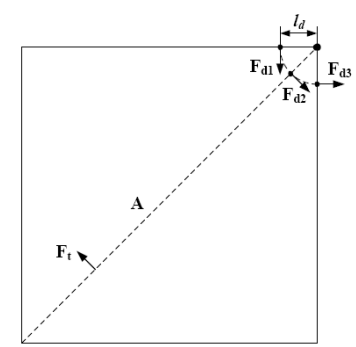

(b) Design domain for one leaf
Fig.5. Design domain for a four-leaf revolving fan

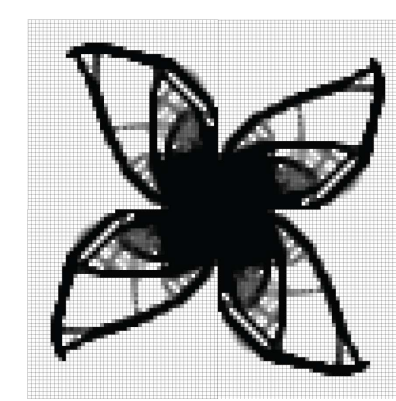

(e) Optimized fan with $\omega=50 \mathrm{rad} / \mathrm{s}$

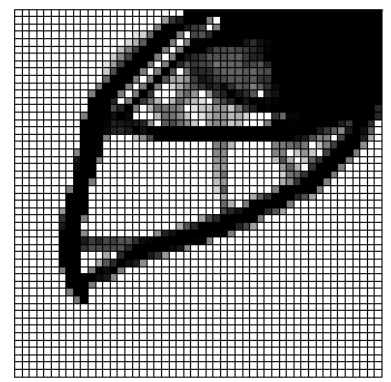

(b) Optimized single leaf with $\omega=50 \mathrm{rad} / \mathrm{s}$

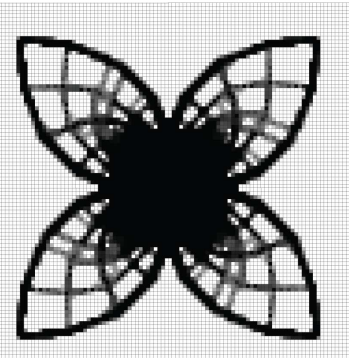

(d) Optimized fan without rotation
Fig.6. Optimized structures for single leaf and fan 
First, a traditional minimizing mean compliance design is performed, no body force is considered in this static case. The final result is shown in Fig.6(a) and a cyclic-symmetric four-leaf structure is assembled in Fig.6(d). Second, the centrifugal force is considered when the fan is rotating at the speed of 50rad/s. The optimized leaf design is shown in Fig.6(b); and the cyclic-symmetric four-leaf fan is assembled and shown in Fig.6(e). Comparing with the previous design, this optimal structure changes the curvatures of upper and lower arches to balance the additional centrifugal force.

The third one is about the design considering the influence of inertia force caused by constant angular acceleration. As shown in Fig.5(b), the design dependent driving force, $\boldsymbol{F}_{d}$, must have the following relation as:

$$
\boldsymbol{F}_{d}=\frac{\boldsymbol{F}_{t} l_{t}+I \alpha}{3 l_{d}}
$$

where $\boldsymbol{F}_{t}$ and $l_{t}$ are the resistance and its moment arm, and both are constant; $l_{d}$ is the moment arm of the driving force. The sensitivity of the design dependent driving force is derived as:

$$
\frac{\partial \boldsymbol{F}_{d}}{\partial x_{i}}=\frac{\alpha}{3 l_{d}} \frac{\partial I}{\partial x_{i}}=\frac{\alpha \rho_{0} v_{i} r_{i}^{2}}{3 l_{d}} .
$$

The predetermined acceleration is chosen as $\alpha=1 \mathrm{rad} / \mathrm{s}^{2}$ at the speed $\omega=0 \mathrm{rad} / \mathrm{s}$. The optimal result in area $A$ and the whole cyclic-symmetric structure are shown in Fig.6(c) and Fig.6(f). Comparing with the first case of this example, we find that a part of material on the leaves distinctly move to the center to decrease the work done by the inertia forces.

\section{Conclusion}

Topology optimization with three types of design dependent body forces, gravitational force, centrifugal force and inertia force, are studied in this paper. Three examples have shown that body forces have strong influences on the final optimal design. We also found that the inequality volume constraint may not be active under the influence of design dependent body forces. It means more material may cause structure weaker when body forces are considered. It is different from the traditional mean compliance design in topology optimization where more material means stronger optimal structure and the inequality volume constraint is always active.

\section{References}

1. M.P. Bendsøe, N. Kikuchi, Generating optimal topologies in structural design using a homogenization method, Computer methods in applied mechanics and engineering, 71, 197-224 (1988)

2. H. Chickermane, H.C. Gea, R.J. Yang, C.H. Chuang, Optimal fastener pattern design considering bearing loads, Struct. Optimi., 17, 140-146 (1999)

3. T. Buhl, Simultaneous topology optimization of structure and supports, Struct. Multidisc. Optim. 23: 336-346 (2002)
4. V.B. Hammer, N. Olhoff, Topology optimization of continuum structures subjected to pressure loading, Struct. Multidisc. Optim. 19, 85-92. (2002)

5. B. Chen, N. Kikuchi, Topology optimization with designdependent loads, Finite Elements in Analysis and Design, 37, 57-70 (2001)

6. M.B. Fuchs, N.N.Y. Shemesh, Density-based topologic design of structures subjected to water pressure using a parametric loading surface, Struct. Multidisc. Optim., 28, 11-19 (2004)

7. B. Zheng, H.C. Gea, Structural topology optimization under design-dependent loads, ASME International Design Engineering Technical Conference, DETC200585605 (2005)

8. O. Sigmund, P.M. Clausen, Topology optimization using a mixed formulation: An alternative way to solve pressure load problems, Computer Methods in Applied Mechanics and Engineering, 196, 1874-1889 (2007)

9. G.I.N. Rozvany, Optimal plastic design:allowance for selfweight, J. Eng. Mech. Div. ASCE 103, 1165-1170 (1977)

10. B.L. Karihaloo, W.S. Hemp, Maximum strength/stiffness design of structural members in presence of self-weight, Proc. R. Soc. Lond. A 389, 119-132 (1983)

11. J.M. Chern, W. Prager, Optimal design of rotating disk for given radial displacement of edge, Jounal of Optimization Theory and Applications, 6(2), 161-170 (1970)

12. J. Huang, G.M. Fadel, Heterogeneous flywheel modeling and optimization, Material and Design 21, 111-125 (2000)

13. S. Turteltaub, P. Washabaugh, Optimal distribution of material properties for an elastic continuum with structure-dependent body force, International Journal of Solids and Structures, 36, 4587-4608 (1999)

14. K. Park, S. Chang, S. Youn, Topology optimization of the primary mirror of a multispectral camera. Struct. Multidisc. Optim. 25(1), 46-53 (2003)

15. M. Bruyneel, P. Duysinx, Note on topology optimization of continuum structures including self-weight, Struct Multidisc Optim 29, 245-256 (2005)

16. H.C. Gea, Topology Optimization: A New MicroStructural Based Design Domain Method, Computers and Structures, 61(5), 781-788 (1996)

17. H. Chickermane, H.C. Gea, Structural optimization using a new local approximation method, Int. J. Numer. Methods Eng. 39(5), 829-846 (1996) 